Proc. Indian Acad. Sci. (Chem. Sci.), Vol. 115, No. 4, August 2003, pp 273-280

(C) Indian Academy of Sciences

\title{
Regioselective photoamination of 4-nitroveratrole upon cyclodextrin complexation
}

\author{
M C DURAI MANICKAM,${ }^{\mathrm{a}} \mathrm{K}$ PITCHUMANI*b and C SRINIVASAN $*^{\mathrm{a}}$ \\ ${ }^{\mathrm{a}}$ Department of Materials Science and ${ }^{\mathrm{b}} \mathrm{S}$ chool of Chemistry, \\ Madurai Kamaraj University, Madurai 625 021, India \\ e-mail: ceesri@yahoo.com
}

MS received 3 March 2003; revised 10 June 2003

\begin{abstract}
Photoamination of 4-nitroveratrole in cyclodextrins with the nucleophiles ammonia, methylamine and hexylamine provides a new route to regioselectivity. This method gives a displaced product para to the nitro group as the predominant product, in contrast to the solution reaction wherein the meta-displaced product predominates. This is due to the change in the mechanistic shift from $S_{N} 2 \mathrm{Ar}^{*}$, wherein the nitro group is meta-directing, to a mechanism involving electron transfer from the nucleophile to the excited aromatic substrate $\left(S_{N}(\mathrm{ET}) \mathrm{Ar} *\right)$ to give the para-displaced product.
\end{abstract}

Keywords. Cyclodextrin; 4-nitroveratrole; photoamination.

\section{Introduction}

Since its first report in 1956, photoinduced nucleophilic aromatic substitution has been the subject of several studies. ${ }^{1}$ While irradiation of nitrobenzene ${ }^{2}$ in liquid ammonia yields about $25 \% p$-nitroaniline and 2\%o-nitroaniline, benzophenone sensitized reaction dramatically improves the yield of $p$-nitroaniline forty-fold. Barltrop and co-workers ${ }^{3}$ have observed that the irradiation of equimolar proportion of anisole and piperidine leads to N-phenylpiperidine in $9 \%$ yield. The photoamination reaction of $m$-nitroanisole ${ }^{4}$ with ammonia yields $90 \% \mathrm{~m}$-nitroaniline and minor amounts of 4-methoxy-2-nitroaniline (2\%) and 2-methoxy-6-nitroaniline $(1 \%)$. This is in contrast to the formation of 2methoxy-4-nitroaniline instead of $m$-nitroaniline in the presence of a forty-fold excess of benzophenone. Thus in the sensitized reaction, substitution takes place at a position para to the nitro group, whereas in the direct irradiation mainly meta- and a small amount of ortho-substitution is observed. These facts indicate that the amination of $m$-nitroanisole proceeds via singlet state in the unsensitized photoamination.

When photoamination of $p$-nitroanisole ${ }^{4}$ is carried out in liquid ammonia or in $15 \%$ ammonia/methanol, the product in both the cases is $p$-anisidine. It has not been established whether this reaction is photosubstitution at the aromatic nucleus or photoreduction of the nitro group by ammonia ${ }^{1}$. Irradiation of $p$-nitroanisole with methylamine $^{5}$, dimethylamine ${ }^{5}$ and ethylamine ${ }^{6}$ gives $\mathrm{N}$-methyl-4-nitroaniline, $\mathrm{N}, \mathrm{N}$ dimethyl-4-nitroaniline and N-ethyl-4-nitroaniline respectively, indicating that amination with alkyl amines results in the substitution of the methoxy group. $p$-Nitroanisole

*For correspondence 
undergoes photoreaction with $n$-hexylamine and ethyl glycinate ${ }^{8}$ giving rise to regioselective photosubstitutions of methoxy and nitro groups respectively. The product of the reaction of $p$-nitroanisole with pyridine in aqueous solution is 1-( $p$-methoxyphenyl)pyridinium nitrite ${ }^{7}$. van Vliet $^{4}$ has studied the photoamination of 3,5-dimethoxynitrobenzene in liquid ammonia and $15 \%$ ammonia in methanol and has observed that the substitution takes place ortho- and meta- to the nitro group. The product ratio in ammonia is $2: 1$, which changes to $10: 1$ in ammonia/methanol.

4-Nitroveratrole in liquid ammonia gives 2-methoxy-5-nitroaniline ${ }^{4}$ in $95 \%$ yield. 4Nitroveratrole also undergoes photosubstitution reactions with primary amines, piperidine and hydroxide ion. ${ }^{9}$ Primary amines and hydroxyl ions cause replacement of the methoxy group in the meta position with respect to the nitro group, whereas piperidine substitutes the methoxy group para to the nitro group in the photo process. Mechanistic evidences indicate that the reaction with piperidine involves a radical ion pair formed via electron transfer from the amine to a triplet state, whereas photoreactions with primary amines involve attack of the amine upon a singlet excited state. These results are justified on the basis of the ionization potential values of the nucleophile and ground state donor-acceptor complex formation abilities.

Cyclodextrins (CDs) are cyclic oligosaccahrides containing six, seven or eight glucose units and are called $\alpha-, \beta$ - and $\gamma$-cyclodextrins respectively. The cavity diameters are approximately $4.5,7.0$ and $8.0 \AA$ for $\alpha-, \beta$ - and $\gamma$-CDs respectively. CDs produce a variety of spectacular complexes with appropriate guest molecules and they are used in catalysis in many reactions. ${ }^{10}$ In their reactions CD complexes mimic enzymes. ${ }^{11}$ The most astonishing feature of $\mathrm{CD}$ is its effective control over the incoming group and its ability to lead to selectivity in a variety of thermal ${ }^{12}$ and photochemical reactions. ${ }^{13}$ This has been exploited to achieve selectivity in photo-Fries rearrangement ${ }^{14}$ and photochemistry of benzoin derivatives. ${ }^{15}$

There is an earlier report by Marquet and co-workers ${ }^{16}$ on the solid state photochemistry of ternary $\beta-\mathrm{CD}$ complexes of 4-nitroveratrole and some nitrophenyl ethers with 1-phenylethylamine. They anticipated that the use of primary amines with a high tendency to proton transfer (following electron transfer step) would translate into a change with respect to the normal behaviour of primary amines and the photoreduction process will dominate. In accordance with their expectation, they report that irradiation of the ternary complexes of $\beta$-CD with several nitrophenyl ethers and 1-phenylethylamine in the solid state produces selective reduction to the corresponding nitroso derivatives.

We have recently reported that photohydroxylation of 4-nitroveratrole in the presence of CDs results in the regioselective formation of 2-methoxy-4-nitrophenol as the exclusive product and this is in contrast to the exclusive formation of 2-methoxy-5nitrophenol in solution photohydroxylation. ${ }^{17}$ This behaviour has been attributed to an increase in the local concentration of the nucleophile upon $\mathrm{CD}$ encapsulation. The active participation of CDs in photohydroxylation causes a shift in reaction mechanism from direct displacement route $\left(S_{N} 2 \mathrm{Ar}^{*}\right)$ to one involving an electron-transfer $\left(S_{N}(\mathrm{ET}) \mathrm{Ar} *\right)$ pathway. The interesting observations of solution reactions of photoamination and photohydroxylation in general and the role of $\mathrm{CD}$ in influencing the mechanism in particular have prompted us to investigate photoamination of $\mathrm{CD}$ complexes of 4nitroveratrole with nucleophiles such as ammonia, methylamine and hexylamine. The results of $\mathrm{CD}$ complexation in photoamination process are presented below. 


\section{Experimental}

\subsection{Materials}

4-Nitroveratrole (Merck), $\alpha$ - and $\gamma$-CDs (American Maize) and $\beta$-CD (Aldrich) were used as received. Methanol (Merck) was doubly distilled and for the amine solution, ammonia (Qualigens), methylamine, hexylamine and benzylamine (Merck) were used as received. For the identification of products, 2-methoxy-4-nitroaniline, 2-methoxy-5nitroaniline, N-methyl-2-methoxy-4-nitroaniline, N-methyl-2-methoxy-5-nitroaniline, $\mathrm{N}$ (1-hexyl)-2-methoxy-4-nitroaniline ${ }^{9}$ and $\mathrm{N}$-(1-hexyl)-2-methoxy-5-nitroaniline ${ }^{9}$ were prepared as reported in literature.

For irradiations a pyrex-filtered, immersion-type photoreactor (Scientific Aids \& Instruments Corp.) fitted with a $400 \mathrm{~W}$ medium pressure mercury vapour lamp with provision for continuous circulation of ice-cold water throughout the experiment was employed. The method of preparation of CD complexes has been described in our earlier paper. $^{17}$

\subsection{Photoamination}

Solid $\alpha$-, $\beta$ - and $\gamma$-CD complexes of 4-nitroveratrole was thoroughly mixed with the ammonia, methylamine or hexylamine solution (15\% of amine solution dissolved in $1: 1$ $\mathrm{MeOH}$-water mixture). This colloidal dispersion after purging with nitrogen was irradiated for $6 \mathrm{~h}$. For the photochemical studies of isotropic solution (1:9 THF-water mixture), $50 \mathrm{mg}$ of substrate was dissolved in $100 \mathrm{ml}$ of amine solution and was irradiated for $6 \mathrm{~h}$ in the same reactor after purging with nitrogen gas for $30 \mathrm{~min}$. The percentage conversion was maintained below $40 \%$ in most cases. Samples were analysed by gas chromatography (Shimadzu 17A) using SE-30 10\% capillary column, FID detector and high purity $\mathrm{N}_{2}$ as carrier gas. In all the GC analyses, retention time of 4nitroveratrole was taken as the internal reference for calibration. Products of the reaction were isolated by column chromatographic separation of the reaction mixture, carried out in bulk quantity. They were identified by GC and also by their ${ }^{1} \mathrm{H}-\mathrm{NMR}$ data $(200 \mathrm{MHz}$, $\left.25^{\circ} \mathrm{C}, \mathrm{TMS}\right)$.

Spectral data of photoproducts: 2-Methoxy-5-nitroaniline (2b) - ${ }^{1} \mathrm{H}-\mathrm{NMR}\left(\mathrm{CDCl}_{3}\right)$ $\delta(\mathrm{ppm}) 7 \cdot 9(d, 1 \mathrm{H}) ; 7 \cdot 7(s, 1 \mathrm{H}) ; 6 \cdot 8(d, 1 \mathrm{H}) ; 3 \cdot 8(s, 3 \mathrm{H})$.

2-Methoxy-4-nitroaniline $(\mathbf{2 c})-{ }^{1} \mathrm{H}-\mathrm{NMR}\left(\mathrm{CDCl}_{3}\right) \delta(\mathrm{ppm}) 7.9(d, 1 \mathrm{H}) ; 7 \cdot 6(s, 1 \mathrm{H}) ; 6.9$ $(d, 1 \mathrm{H}) ; 3 \cdot 6(s, 3 \mathrm{H})$.

2-Methoxy-5-nitro-N-methylaniline $(3 \mathbf{b})-{ }^{1} \mathrm{H}-\mathrm{NMR}\left(\mathrm{CDCl}_{3}\right) \delta(\mathrm{ppm}) 8 \cdot 0(d, 1 \mathrm{H}) ; 7 \cdot 8(s$, $1 \mathrm{H}) ; 7 \cdot 0(d, 1 \mathrm{H}) ; 4 \cdot 0(s, 3 \mathrm{H}) ; 2 \cdot 0(s, 3 \mathrm{H})$.

2-Methoxy-4-nitro-N-methylaniline $(3 \mathrm{c})-{ }^{1} \mathrm{H}-\mathrm{NMR}\left(\mathrm{CDCl}_{3}\right) \delta(\mathrm{ppm}) 7 \cdot 9(d, 1 \mathrm{H}) ; 7 \cdot 6(s$, $1 \mathrm{H}) ; 6 \cdot 8(d, 1 \mathrm{H}) ; 3 \cdot 9(s, 3 \mathrm{H}) ; 3 \cdot 0(s, 3 \mathrm{H})$.

(4b) and (4c) are identified by GC-MS (Finnigan GC-MS with RTX5-MS capillary column and high purity helium as carrier gas) and also by analogy of their retention times with those of $(\mathbf{2 b})-(\mathbf{3 c})$. 
2-Methoxy-4-nitro-N-hexylaniline (4b) - retention time $(15.03 \mathrm{~min}) ; \mathrm{m} / \mathrm{e}$ values: 252 (M I), 181 (base peak), 166, 135.

2-Methoxy-5-nitro-N-hexylaniline $(4 \mathrm{c})$ - retention time $(14.45 \mathrm{~min}) ; \mathrm{m} / \mathrm{e}$ values: 252 (M I), 181 (base peak), 152, 166, 135.

The complex formation of the substrate was confirmed as described earlier ${ }^{17}$ by monitoring the changes in absorption maximum $(340 \mathrm{~nm})$ upon addition of varying concentration of CDs. It is relevant to note that addition of CD does not affect the $\lambda_{\max }$, though the $\varepsilon_{\max }$ increases progressively. It is also confirmed, by independent studies, that the addition of nucleophiles (2)-(4) to the host-guest system does not affect either the $\lambda_{\max }$ or the $\varepsilon_{\max }$ with all the three CDs.

As the conversions are efficient under the present experimental conditions, quantum yield of the reaction was not calculated. Control experiments show that the products are photostable under the present reaction conditions. Also, in most of the CD-mediated reactions, the percentage conversions are restricted to $<50 \%$. All these exclude any possible effect of photoproducts on the course of the reaction.

\section{Results and discussion}

Photoamination of 4-nitroveratrole (1) with ammonia (2), methylamine (3) and hexylamine (4) were carried out in isotropic medium and also as their complexes with $\alpha$-, $\beta$ - and $\gamma$-CDs. The products distribution and the percentage yield of the various products are presented in table 1 . Photoamination of $\mathbf{1}$ in THF-water (1:9) with $15 \%$ of 2 gives only $\mathbf{2 b}$ in which the methoxy group meta- to the nitro group is displaced (scheme 1). However, when photolysed as $\alpha$-, $\beta$ - and $\gamma$-CD complexes of 1 in $1: 1 \mathrm{MeOH}$-water

Table 1. Products distribution in photoamination* of 1 with 2,3 and 4.

\begin{tabular}{lcccc}
\hline & & \multicolumn{3}{c}{ Percentage of } \\
Nucleophile & Medium & $\mathbf{1}$ & $\mathbf{b}^{* *}$ & $\mathbf{c}^{* *}$ \\
\hline $\mathbf{2}$ & THF-water $(1: 9)$ & 73 & 27 & - \\
& $\alpha-C D$ & 93 & - & 7 \\
& $\beta-C D$ & 79 & - & 21 \\
$\mathbf{3}$ & $\gamma$-CD & 78 & - & 22 \\
& THF-water $(1: 9)$ & 79 & 19 & 2 \\
& $\alpha-C D$ & 73 & 12 & 15 \\
& $\beta-C D$ & 62 & 17 & 21 \\
4 & $\gamma-C D$ & 51 & 3 & 46 \\
& THF-water $(1: 9)$ & 20 & 65 & 15 \\
& $\alpha-C D$ & 40 & 20 & 40 \\
& $\beta-C D$ & 38 & 12 & 50 \\
& $\gamma$-CD & 75 & - & 25 \\
\hline
\end{tabular}

*Irradiated for $6 \mathrm{~h}$; $\mathrm{CD}$ complexes were irradiated in $1: \mathrm{MeOH}$-water mixture; Products were analysed by GC; error limit $\pm 2 \%$

**For structures of $\mathbf{b} \& \mathbf{c}$, see scheme 1 
<smiles>COc1ccc([N+](=O)[O-])cc1OC</smiles>

(1)<smiles>[R]Nc1ccc([N+](=O)[O-])cc1OC</smiles>

(2b) (2c)

(4c)

Scheme 1. Structures of the various products formed in the photoamination of 1 with amine nucleophiles.

mixture of 2, a remarkable alteration in selectivity is observed. Isomeric $\mathbf{2 c}$, in which the methoxy group para- to the nitro group is displaced, has been obtained as the exclusive product.

Photoamination of $\mathbf{1}$ in solution (table 1 ) with $15 \%$ of $\mathbf{3}$ yields a mixture $\mathbf{3 b}$ and $\mathbf{3 c}$ (meta- and para-substituted products respectively), of which $\mathbf{3 b}$ is the major product (table 1). This is in accordance with earlier reports. ${ }^{9}$ However, photoamination of $\mathbf{1}$ as its $\mathrm{CD}$ complex with $\mathbf{3}$ in solution yields the para-substituted compound $\mathbf{3 c}$ as the major product. As the size of the cavity increases from $\alpha$ - to $\gamma-\mathrm{CD}$, the percentage of $\mathbf{3 c}$ has progressively increased.

In the solution photolysis of $\mathbf{1}$ in the presence of $15 \%$ of $\mathbf{4}$, both meta- and paramethoxy displaced products $(\mathbf{4 b}$ and $\mathbf{4 c}$ ) are observed, in which $\mathbf{4 b}$ is the major product. However, when $\alpha-, \beta$ - and $\gamma$-CD complexes of $\mathbf{1}$ are photolysed with $\mathbf{4}$, a change in the selectivity is observed in which isomeric $\mathbf{4} \mathbf{c}$ is obtained as the major product. Here also, as in the previous cases, an increase in the cavity size causes an increase in the yield of $\mathbf{4 c}$ at the expense of $\mathbf{4 b}$ and with $\gamma$-CD complex $\mathbf{4} \mathbf{c}$ is the exclusive product. We have not observed the formation of nitroso compounds during the photolysis of $\mathrm{CD}$ complexes of 1 and this is in contrast to the selective photoreduction to the corresponding nitroso derivative on irradiation of the ternary of complex of $\beta-\mathrm{CD}, \mathbf{1}$ and 1-phenylethylamine in the solid state. ${ }^{16}$

Three kinds of mechanisms have been proposed for aromatic nucleophilic photosubstitution. ${ }^{18}$ This has been elaborated in detail in our recent paper $^{17}$ on photohydroxylation of 1 . In a direct displacement $\left(S_{N} 2 \mathrm{Ar}^{*}\right)$ route, in which the nucleophile adds onto the photoexcited substrate in its triplet state, the dominant interaction is between the HOMO of the substrate and the HOMO of the nucleophile. ${ }^{19}$ The nucleophile now goes to the position meta- to the nitro group and the regioselectivity is controlled by the size of the energy gap between the excited state nucleophile encounter complex and the ground state $\sigma$-complex. Another mechanistic pathway involves electron-transfer from the nucleophile to the excited aromatic substrate $\left(S_{N}(\mathrm{ET}) \mathrm{Ar}^{*}\right){ }^{20}$ Here the dominant interaction is between the LUMO of the aromatic substrate and the singly occupied HOMO of the nucleophile. ${ }^{19}$ In this type of interaction, replacement of substitutents para- to the nitro group takes place. Studies on photo-Smiles rearrangement provide evidence for this type of pathway. ${ }^{21}$ The third mechanism involves an electron transfer from the aromatic substrate to an acceptor, followed by attack of the nucleophile on the aromatic radical cation $\left(S_{R-N} 1 \mathrm{Ar} *\right)$. 
Further, regioselectivity also depends on the nature of nucleophiles, particularly on their ionization potential in the case of amines. With the three amines used in the present study, the ionization potential increases in the order $\mathrm{NH}_{3}<\mathrm{CH}_{3} \mathrm{NH}_{2}<\mathrm{C}_{6} \mathrm{H}_{13}-\mathrm{NH}_{2}$. When $\mathbf{1}$ is photoaminated in solution $\mathbf{2 b}, \mathbf{3 b}$ and $\mathbf{4 b}$ are formed as the predominant products. This is in accordance with the expected regioselectivity (meta-substitution) and $S_{N} 2 \mathrm{Ar}^{*}$ mechanism operating in this situation. With amine nucleophiles $\mathbf{3}$ and $\mathbf{4}$, a minor contribution of electron-transfer $\left(S_{N}(\mathrm{ET}) \mathrm{Ar}^{*}\right.$ mechanism) is evident from formation of 3c and $\mathbf{4 c}$. It is interesting to note that this is in contrast to photohydroxylation, wherein solution photolysis of 4-nitroveratrole does not yield any para-substituted product. ${ }^{17}$

However, when $\alpha-, \beta$ - and $\gamma$-CD complexes of $\mathbf{1}$ are irradiated in the presence of $\mathbf{2 , 3}$ and $\mathbf{4}$, isomeric $\mathbf{2 c}, \mathbf{3 c}$ and $\mathbf{4 c}$ products formed by the displacement of methoxy group para- to the nitro group are obtained as the major products. We propose that upon CD encapsulation, a mechanistic changeover takes place and $S_{N}($ ET)Ar* pathway is the predominant mechanism now. This is attributed to the better electron-donor capabilities of amines, which facilitates electron transfer, when they are held in close contact with the substrate in a ternary complex (scheme 2). This situation is different from solution photolysis, where the substrate and nucleophile are freely moving. As a consequence of this mechanistic shift, the methoxy group, which is para- to the nitro group is displaced readily (scheme 2 ).

Additional support for the observed results comes from orbital considerations. In solution photoamination which is HOMO controlled and the operating mechanism is $S_{N} 2 \mathrm{Ar}^{*}$, activation and meta-direction by nitro groups is well-established and accordingly $\mathbf{2 b}, \mathbf{3 b}$ and $\mathbf{4 b}$ are the exclusive/major products. Charge densities (scheme 3) calculated

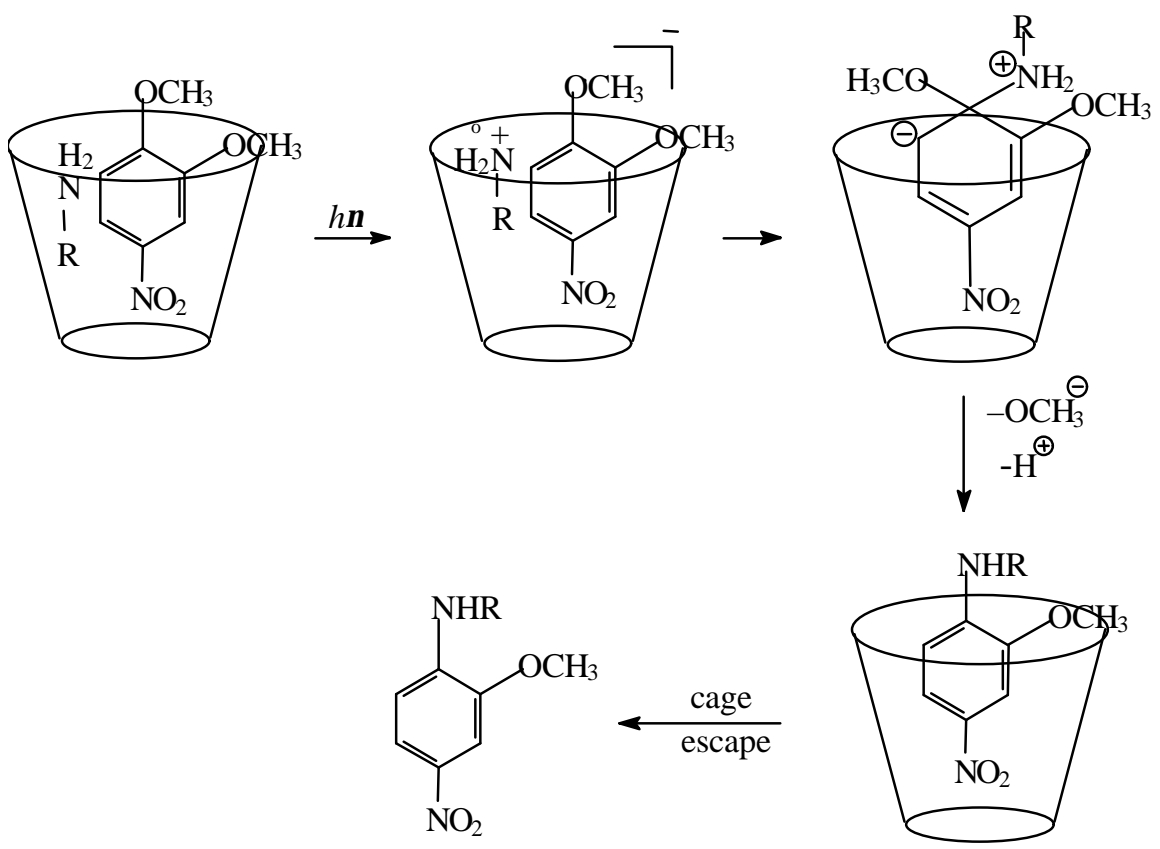

Scheme 2. Mechanism of photoamination of 1 upon CD complexation. 


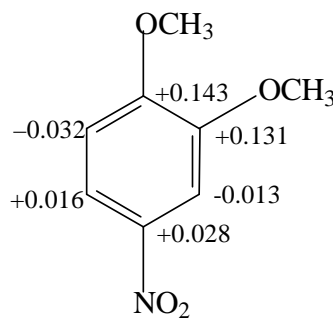

$S_{0}$

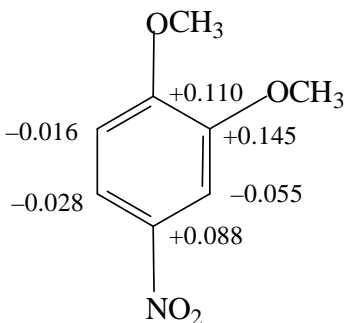

$T_{1}$

Scheme 3. Calculated charge densities for the various positions of $\mathbf{1}$ in the ground and lowest triplet states.

for the various positions of $\mathbf{1}$ in the ground and lowest triplet states (CNDO/2 method) reveal the qualitative correlation with the observed reactivity. Positions with larger positive charge show higher reactivity. A ground state mesomeric interaction between para-methoxy and nitro groups as shown in scheme 4 leaves the para-position less vulnerable for nucleophilic attack, despite its having a slightly higher positive value in $S_{o}$ state.

However, upon $\mathrm{CD}$ complexation of 4-nitroveratrole, the mechanistic changeover to $S_{N}($ ET)Ar* makes the reaction now controlled by the dominant interaction between the LUMO of the substrate and the HOMO of the nucleophile and a reactivity analogous to ground state reactivity prevails. The activating and ortho-/para-directing nature of the methoxy group (which dominates the deactivating and meta directing nitro group) controls the combination of 4-nitroveratrole radical anion with the amine radical cation to form a $\sigma$ complex. It is also likely that a simplistic explanation based on frontier orbital may not be sufficient for $S_{N}(\mathrm{ET}) \mathrm{Ar} *$ mechanism as, after electron transfer, energies, coefficients and charges will be very different from the ground state.

In addition, steric effects (which accrue upon CD complexation) may also play a significant role. Upon inclusion into $C D$ cavity the para-methoxy group of $\mathbf{1}$ is more exposed (scheme 2) and there is also significant steric inhibition of ground state mesomeric interaction between the para-methoxy and nitro groups visualised earlier, thus leading to predominant formation of $\mathbf{2 c}$.

Two important features are noticed from the results obtained in the present study: (a) with stronger electron-donors methylamine and hexylamine, para-substituted products are also obtained even in the absence of CDs, emphasizing the significance of electrontransfer pathway in photoamination compared to photohydroxylation, ${ }^{17}$ upon CD complexation, the percentage of para-substituted products $(\mathbf{2 c}, \mathbf{3 c}$ and $\mathbf{4 c})$ increases, while that of $\mathbf{2 b}, \mathbf{3 b}$ and $\mathbf{4 b}$ decreases. This effect is more pronounced as the cavity size increases from $\alpha$ - to $\gamma$-CD. Thus CD complexation, which holds together both the amine nucleophile and the substrate in closer proximity, promotes electron-transfer more efficiently than in its absence. (b) Side reactions ${ }^{16,22,23}$ such as photoreduction (to yield nitroso compounds, which can undergo subsequent coupling to give azoxy as well as azo derivatives) when nitro compounds are irradiated in the presence of amine nucleophiles are not observed under the present experimental conditions.

Photoinduced nucleophilic substitution of 1 with benzylamine (5) in the presence of $\mathrm{CD}$ resulted in the dominant formation of benzylamine-derived complex products. This is 


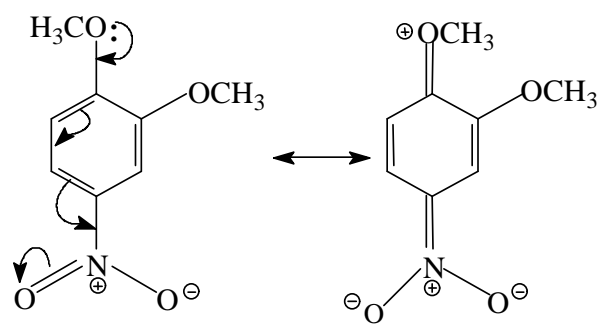

Scheme 4. Mesomeric interaction between para-methoxy and nitro groups.

probably due to the inclusion of $\mathbf{5}$ into the $\mathrm{CD}$ cavity in preference to the trisubstituted aryl ring of 4-nitroveratole.

\section{Conclusion}

CD complexation of 4-nitroveratrole brings about a remarkable shift in mechanistic pathway (from a direct displacement path to one involving electron-transfer) and consequent alteration in regioselectivity. Photoamination involving an electron-transfer pathway is observed with amine nucleophiles, upon $\mathrm{CD}$ complexation because the nucleophile and the substrate are in close proximity. In addition, other side reactions such as photoreduction and coupling etc., are totally suppressed. The present study is an elegant demonstration of the usefulness of CDs as ideal hosts for exhibiting remarkable control over the photochemical reactivities of the included guests.

\section{References}

1. Cornelisse J and Havinga E 1975 Chem. Rev. 75353

2. van Vliet A, Kronenberg M E and Havinga E 1966 Tetrahedron Lett. 75957

3. Barltrop J A, Bunce N J and Thomson A 1967 J. Chem. Soc. C 1142

4. van Vliet A, Kronenberg M E, Cornelisse J and Havinga E 1970 Tetrahedron 261061

5. Kronenberg M E, van der Heyden A and Havinga E 1966 Rec. Trav. Chim. Pays-Bas 8556

6. McCain J H 1967 Ph D thesis, Evanston, Il

7. Letsinger R L, Ramsay O B and McCain J H 1965 J. Am. Chem. Soc. 872945

8. Cantos A, Marquet J and Moreno-Manas M 1989 Tetrahedron Lett. 302423

9. Cantos A, Marquet J, Moreno-Manas M and Castello A 1988 Tetrahedron 442607

10. Takahashi K 1998 Chem. Rev. 982013

11. Saenger W 1980 Angew. Chem., Int. Ed. Engl. 19344

12. Pitchumani K, Velusamy P and Srinivasan C 1994 Tetrahedron 507903

13. Pitchumani K, Devanathan S and Ramamurthy V 1992 J. Photochem. Photobiol. A69 201

14. Pitchumani K, Durai Manickam M C and Srinivasan C 1991 Tetrahedron Lett. 322975

15. Syamala M S and Ramamurthy V 1988 Tetrahedron 447223

16. Mir M, Marquet J and Cayon E 1992 Tetrahedron Lett. 467053

17. Durai Manickam M C, Pitchumani K and Srinivasan C 2002 J. Photochem. Photobiol. A149 131

18. van Riel H A H C, Lodder G and Havinga E $1981 \mathrm{~J}$. Am. Chem. Soc. 1037257

19. Havinga E and de Jong R O 1962 Bull. Soc. Chim. Belg. 71803

20. van Vliet A, Cornelisse J and Havinga E 1969 Rec. Trav. Chim. Pays-Bas 881339

21. Mutai K, Nakagaki R and Tukada H 1985 Bull. Chem. Soc. Jpn. 532472

22. Dopp D 1975 Top. Curr. Chem. 5549

23. Sundararajan K, Ramakrishnan V and Kuriacose J C 1983 Indian J. Chem. B22 257 